\section{Radioactive isotopes: astrophysics answers in abundance}

Dr Benjamin Wehmeyer Konkoly Observatory, Hungary and University of Hertfordshire, UK Professor Dr Chiaki Kobayashi University of Hertfordshire, UK. Dr Maria Lugaro

Konkoly Observatory, Hungary

How were the elements which we are made of formed?

How can meteorites and deep-sea sediments help us better understand nuclear processes and propagation phenomena in the galaxy?

How can they help us understand if our solar system is unique or if it has many siblings?
Shortly after the Big Bang, the first nuclei were formed: hydrogen, helium, and some traces of lithium. These elements were abundant in gas clouds. After a while, the first stars formed out of this gas. Stars are basically giant gas balls. The gravity of such a gas ball increases the pressure in its centre, which becomes so high that nuclear hydrosen fusion is so high that nuclear hydrogen fusion is ignited. This nuclear hydrogen fusion then releases heat which counteracts the further gravitational collapse of the star. The fusion in the star fuses the hydrogen to helium until enough helium has been produced in the star to ignite the nuclear helium as well.

For intermediate-mass stars like our Sun, this is the last burning stage before they eject their outer envelopes into the galaxy and their cores cool down as white dwarfs. However, massive stars can ignite further nuclear burning stages until they produce nickel and iron. Beyond nickel and iron, nuclear fusion requires energy, so the nuclear burning in the centre of a massive star cannot contribute to withstanding the gravitional collapse of the ster the gravit The massive star collapes under its weight and explodes in a supernova; ejecting its outer shells while leaving a neutron star behind.

If we look at a binary massive star system, both stars might undergo a supernova explosion leaving two neutron stars behind. If their distance and initial momentum are suitable, the can orbit each other and slowly spira inwards towards their common centre of mass under the emission of gravitationa waves. When they collide in a so-called neutron star merger, some of the materia of the two neutron stars will experience a. strong neutron density, which wil force the nuclei to become very neutron rich. Some of these very neutron-rich nuclei can be ejected from the neutron star merger and decay towards stability. This decay is actually a $B$-decay, which means that the excess neutrons are converted into protons. More protons in a nucleus mean that, in general, a heavier

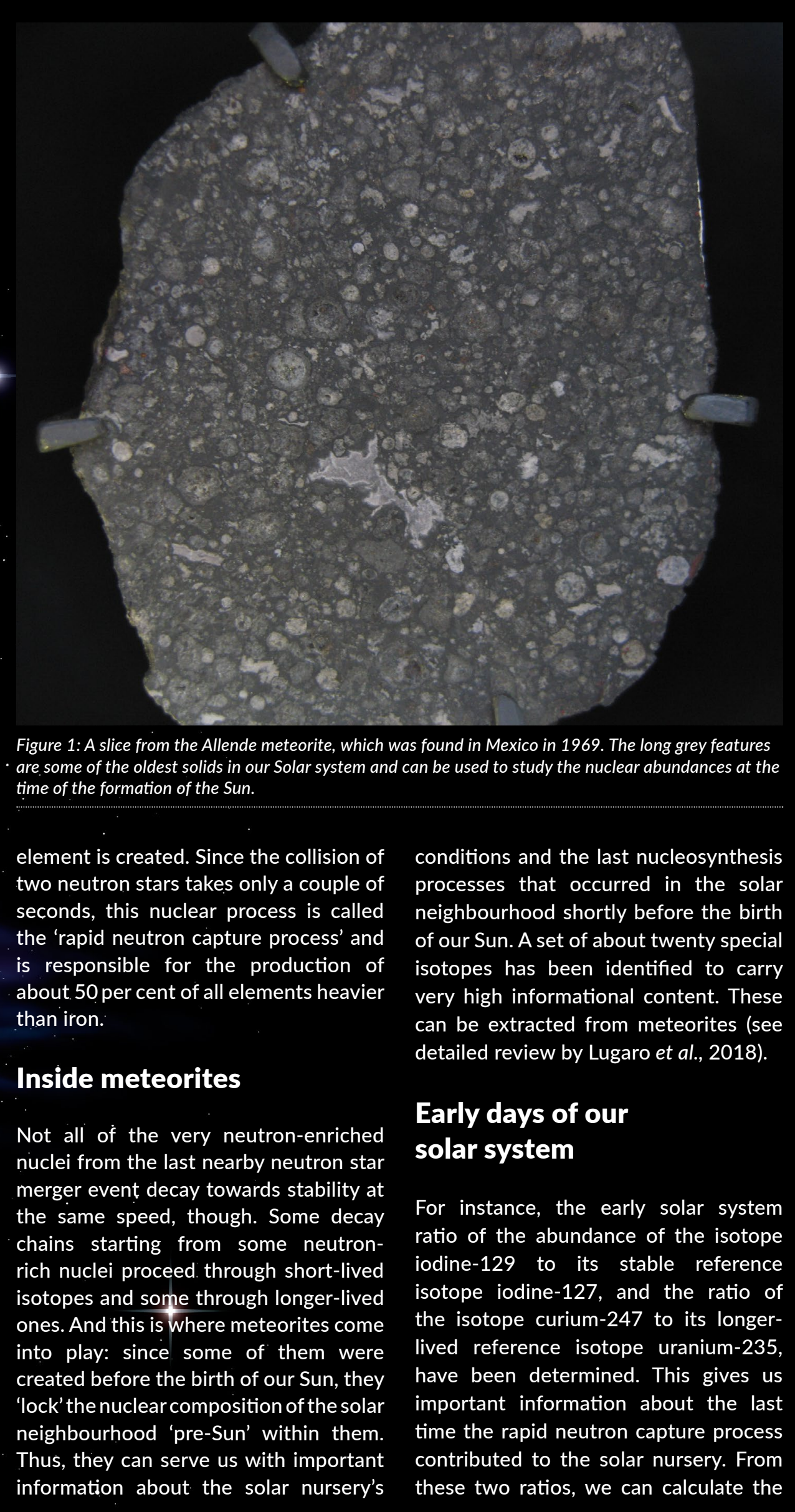




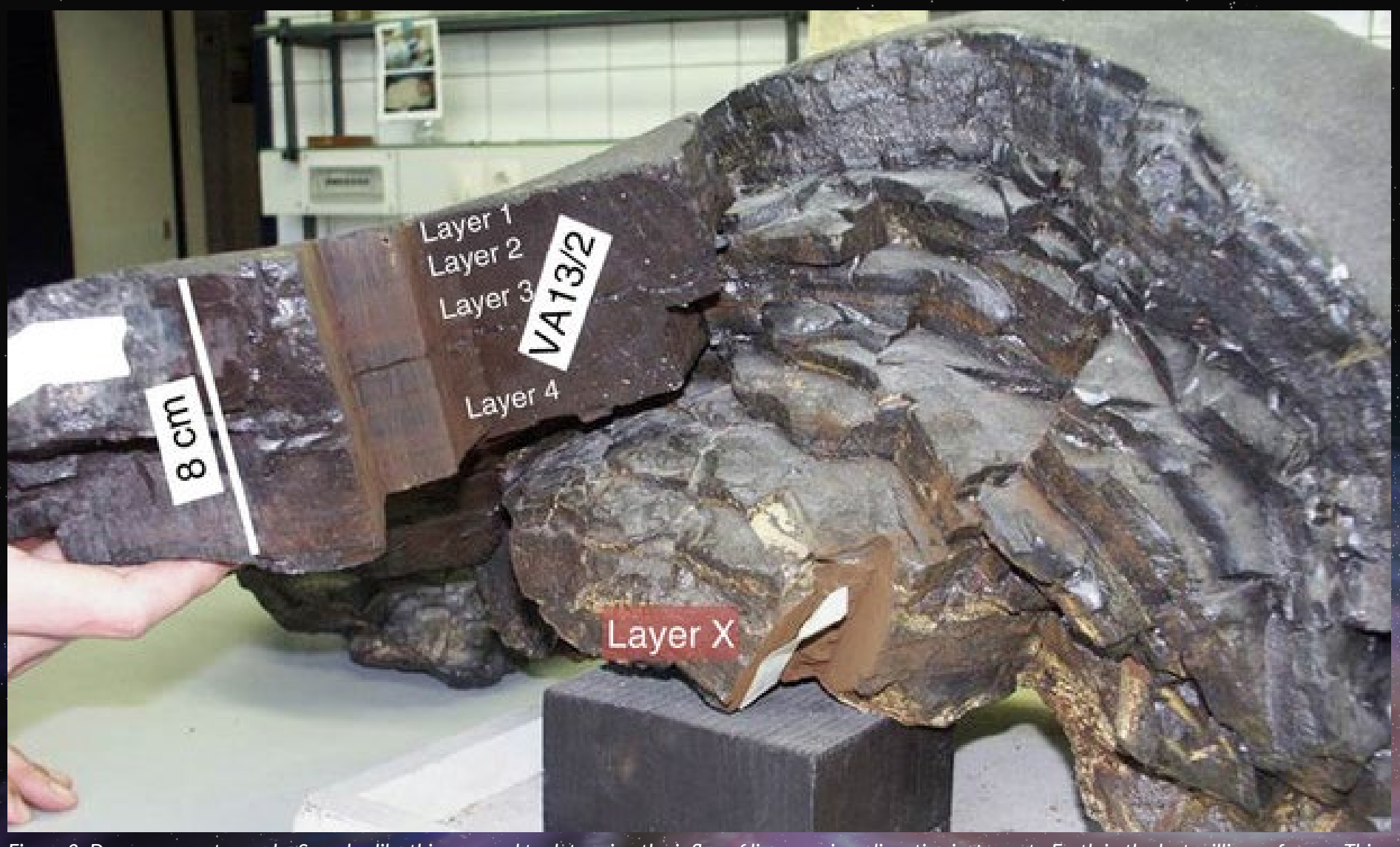

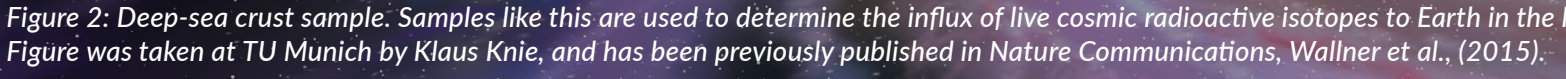

abundance ratio of iodine-129 and maybe the nucleosynthesis period curium-247 in the early solar system, during the neutron star merger was too two isotopes that can be directly short, or maybe the model assumed too produced in neutron star mergers. Since much fission of heavier isotopes causing the two isotope hive very simita halflives, their nuclear abundace rio the early solar system allows us to draw conclusions about their ratio at the time of their production in the last neutron star merger that contributed to the solar nursery. This can help us directly determine at which astrophysica conditions the last neutrơn star merge occurred (Côté et al., 2021). This result also very important for both the nuclear physics and the high energy physics community. Since they now know the ratio at which these two distinct isotopes were produced the last time before the formation of our Sun they can use that information to benchmark. their modelling and their simulations that ratio. For instance, if a simulation of the rapid neutron capture primulation a neutron star merger yields an excess a neutron star merger yields an excess
of the much lighter iodine-129 in comparison to the heavier çurium-247, thought to be produced in supernovae, nd plutonium-244 is thought to be exclusively produced in neutron star. mergers. Suppose we combine that information with the data about these two isotopes, which we already have from meteorites. In that case,-we can conclude that the solar neighbourhood

\section{Back on Earth}

Another very interesting insight radioactive nuclei can provide comes from their ongoing deposition on Earth. Wallner et al. (2021) have selected excavated samples from the sea-floor. iron-60 and plutonium-244 isotopes at different depths in the samples. With their modelling of the accretion rate of sediments to the deep-sea floor and a prescription for the influx of these two isotopes into the solar system and onto Earth, they were able to derive the atomic density of these two isotopes in the solar neighbourhood over the last ten million years. These two isotopes are so interesting because iron- 60 is multiple areas in the ocean and They discovered multiple radioactive dispersion effects have brought these isotopes closer to the Sun, or maybe nearby supernovae have carried them

\section{The Milky Way}

A third lesson to be learned from using radioactive isotopes for astrophysics is how prevalent they are throughout the galaxy and if they are evenly distributed. We use sophisticated computer programs to simulate the formation of the Milky Way galaxy in great detail, (e.g. Vincenzo and Kobayashi, 2020). Among many other features we give birth to stars and let them die, let them produce elements and explode them, so they distribute some of them, so they disto some of the elements that they produced during We monitor the gas in the galaxy and We monitor the gas in the galaxy and galaxy has evolved for 9 billion years galaxy has evolved for 9 bition years (4.5 bilion years ago-the time of the solar system's bith), we analyse its contents. We can see exactly where in
the galaxy which density of the isotopes of interest is present.

\section{Chances of life}

The simulations allow us to determine whether the amounts of those isotopes in meteorites represent normal atomic densities of these isotopes or, as in the

majority of space in the galaxy, if the nsity of these isotopes was higher or ower. Since some of the isotopes we focus on are also highly relevant for the heating of planetesimals (Lichtenberg et al., 2019), they are also important for the development of those planetesimals and or later maintaining a temperature that permits liquid wategr. Thus, some of the sotopes in question are also relevant for any form of life in any stellar system. If the meteoritic data are an outlier in the results of the galaxy simulation, we can speculate whether we are the only stellar system capable of hosting life in the galaxy. If the data fall in the bulk of the results of the galaxy simulation, many solar-like systems can be expected our galaxy.

The simulation snapshots provide us with precise information on where exactly in the galaxy to expect such otope densities. This allows us to further constrain which regions of our galaxy could potentially host lif. Follow-up observations could then b. carried out and checked to see whether these isotopices are actually abundant in these regions. If, for instance, it found in our simulations that solar system-like isotope densities are to be found in the centre of our galaxy's spira arms, telescopes could be pointed in those directions to seek other signs of extraterrestrial life.

\section{References}

Côté, B., Eichler, M., Yagüe López, A., Vassh, N., Mumpower, M.R., Világos, B., Soós, B., Arcones,
A., Sprouse, T.M., Surman, R., Pignatari, M., Petó, M.K., Wehmeyer, B., Rauscher, Th. and Lugaro A., Sprouse, T.M. Surman, R., Pignatari, M., Petó, M.K., Wehmeyer, B., Rauscher, Th. and Lugaro,
M. (2021) '1291 -and $247 \mathrm{Cm}$ in meteorites constrain the last astrophysical source of solar
r-process elements', Science, 371(16532), pp. $945-948$.

Lichtenberg, T., Golabbek, G.J., Burn, R., Meyer, M.R, Alibert, Y., Gerya, T. V. and Mordasini; C.
(2019) 'A water budget dichotomy of rocky protoplanets from 26Al-heating', Nature Astronom 3; pp. 307-313

Lugaro, M., Ott, U. and Kereszturi, A.' (2018) 'Radioactive nuclei from cosmochronology to
habitability', Progress in Particle and Nuclear Physics, 102, pp.1-47. habitability, Progress in Particle and Nuclear Physics, 102, pp. 1-47.

Vincenzo, F., Kobayashi, C., (2020) 'Stellar migrations and metal flows - Chemical evolution of the thin 'disc of a simulated Milky Way analogous galaxy', Monthly Notices of the Royal Astronomic
Society, Volume 496 , Issue 1, pp. 80-94, dai 10.1093/mnras/sto 1451. Wallner, A. Faestermann, T., Feige, J. et al. (2015)'Abundance of Wallner, A., Faestermann, T., Feige, J. et al. (2015)'Abundance of live 244Pu in deep-sea
reservoirs on Earth points to rarity of actinide nucleosynthesis', Nature Communications, 6.5956 reservoirs on Earth points to
doi: $10.1038 /$ ncomms

Wallner, A., Froehlich, M.B., Hotchkis, M.A.C., Kinoshita, N., Paul, M., Martschini, M., Pavetich,
S., Tims, SG. Kivel N. Schumann, D. Honda: M. Matsuzaki. H. and Yamagata. T. (2021) '60F s.nd 244Pu deposited on Earth constrain the r-process vields of recent nearby supernova

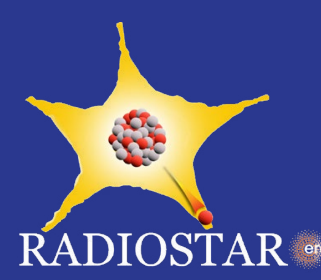

PROJECT SUMMARY

The ERC Consolidator Grant project "RADIOSTAR: Radioactivities form Stars to Solar Systems" uses radioactive nuclei produced by nuclear reactions inside stars and supernovae to understand the history of the chemical matter that builds up our Sun, our planet and ourselves, enerating a new understanding of our solar system and the life within it.

\section{PROJECT LEAD}

This sub-project of RADIOSTAR is led by $\mathrm{Dr}$ Benjamin Wehmeyer. Born in Westerland Sylt (Germany), he received his PhD from the University of Basel (Switzerland) in 2016. He USA) for to North Carolina State Universty USA) for a postdoctoral research posstion fellowship of the Swiss National Science Foundation.

In 2019, he joined the RADIOSTAR project to work with DrLugaro at the Konkoly Observatory University of Hertfordshire, UK

\section{PROJECT PARTNERS}

The RADIOSTAR project (PI Dr Maria Lugaro) is based at the Konkoly Observatory in Budapest, part of the multidisciplinary Research Centre for Astronomy and Earth Sciences of the Hungarian developed in collaboration with Prof. Dr Chiaki Kobayashi at the University of Hertfordshire, UK.

\section{CONTACT DETAILS}

Benjamin Wehmeye

1 Budapest, Hungary, Konkoly-Thege Miklós út 15-17.

푤 +3613919363

崩enjamin.wehmeyer@csfk.org www.konkoly.hu/radiostar

Pl: Maria Lugaro

$\square$ maria.lugaro@csfk.org

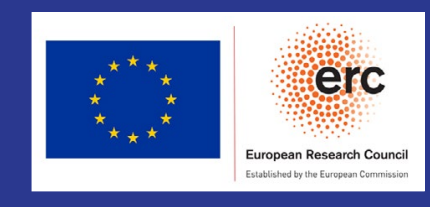

FUNDING

This project has received funding from the European the European Unions
innovation programme under grant agreement No.724560. 